\title{
Methamphetamine Activates Reward Circuitry in Drug Naïve Human Subjects
}

\author{
Birgit A Völlm*,', Ivan E de Araujo ${ }^{2}$, Philip J Cowen', Edmund T Rolls ${ }^{2}$, Morten L Kringelbach ${ }^{3}$, \\ Katharine A Smith', Peter Jezzard ${ }^{3}$, Ronald J Heal ${ }^{3}$ and Paul M Matthews ${ }^{3}$ \\ 'Department of Psychiatry, University of Oxford, Warneford Hospital, Oxford, UK; ${ }^{2}$ Department of Experimental Psychology, University of \\ Oxford, South Parks Road, Oxford, UK; ${ }^{3}$ Centre for Functional Magnetic Resonance Imaging of the Brain, John Radcliffe Hospital, Oxford, UK
}

\begin{abstract}
Amphetamines are highly addictive drugs that have pronounced effects on emotional and cognitive behavior in humans. These effects are mediated through their potent dopaminergic agonistic properties. Dopamine has also been implicated in the modulation of responses of the 'reward circuit' in animal and human studies. In this study we use functional magnetic resonance imaging (fMRI) to identify the brain circuitry involved in the psychostimulant effect of methamphetamine in psychostimulant-naiive human subjects. Seven healthy volunteers were scanned in a 3T MR imaging system. They received single-blind intravenous infusions of methamphetamine $(0.15 \mathrm{mg} / \mathrm{kg})$, and rated their experience of 'mind-racing' on a button press throughout the experiment. Data were analyzed with statistical parametric mapping methods. Amphetamine administration activated the medial orbitofrontal cortex, the rostral part of the anterior cingulate cortex, and the ventral striatum. Ratings of 'mind-racing' after methamphetamine infusion correlated with activations in the rostral part of the anterior cingulate cortex and in the ventral striatum. In addition, activations in the medial orbitofrontal cortex were independent of motor and related responses involved in making the ratings. These findings indicate that the first administration of a psychostimulant to human subjects activates classical reward circuitry. Our data also support recent hypotheses suggesting a central role for the orbitofrontal cortex in drug reinforcement and the development of addiction.

Neuropsychopharmacology (2004) 29, I7I5-1722, advance online publication, 12 May 2004; doi: I 0. I 038/sj.npp. I 30048 I
\end{abstract}

Keywords: amphetamine; human; $\mathrm{fMRl}$; orbitofrontal cortex; psychological effect; dopamine

\section{INTRODUCTION}

Amphetamines are widely abused for their stimulant and euphoriant properties (Murray, 1998). In particular, the methylated derivative, methamphetamine, is a highly addictive drug known for its potent reinforcing effects, as observed in monkeys (Woolverton et al, 1984) and humans (Volkow et al, 2001). Animal and human studies suggest that these effects are mediated through activation of the mesolimbic dopamine system, which innervates limbic and cortical areas including the ventral striatum, orbitofrontal cortex, and anterior cingulate cortex to form a 'reward circuit' (Rolls, 1999; Goldstein and Volkow, 2002).

Within this reward circuitry, a critical role would appear to be played by the orbitofrontal cortex. This region has been shown to be centrally involved in the representation of primary and secondary reinforcers in the primate brain,

\footnotetext{
*Correspondence: BA Völlm, Neuroscience and Psychiatry Unit, University of Manchester, Stopford Building, Oxford Rd, Manchester MI3 9PT, UK, Tel: + 44 I6I 275 7670, Fax: + 44 I6I 2757429 , E-mail: birgit.vollm@man.ac.uk

Received 22 September 2003; revised 08 March 2004; accepted 12 March 2004

Online publication: 18 March 2004 at http://www.acnp.org/citations/ Npp031804030434/default.pdf
}

including humans (Rolls, 1999, 2000). It has been shown that macaques will work to self-administer D-amphetamine into the orbitofrontal cortex (Phillips and Rolls, 1981). In humans, abnormal metabolic activity and low levels of $D_{2}$ dopamine receptor availability have been observed in the orbitofrontal cortex of methamphetamine abusers (Volkow et al, 2001), which confirms analogous findings in cocaine, alcohol, and heroin abusers and leads to the hypothesis that disruption of (orbito)frontal cortical circuits may lead to compulsive drug intake (Volkow and Fowler, 2000; Volkow et al, 2001; Goldstein and Volkow, 2002).

Although the role of reward-related circuitries in addiction has been previously explored by applying functional imaging to study the responses to drug administration in the brains of people with substance abuse disorders (eg Breiter et al, 1997), relatively little is known about the responses in these brain regions to drug administration during the initial stages of addiction or in the case of drugnaïve subjects.

In this study we use functional magnetic resonance imaging (fMRI), a noninvasive functional brain mapping technique with high spatial and temporal resolution, to demonstrate that intravenous methamphetamine administered to drug-naive subjects produces limbic and cortical activations consistent with postulated reward circuitry, in 
particular in the orbitofrontal cortex. These activations correlate with behavioral ratings reflecting the subjective effects produced by methamphetamine administration.

\section{MATERIALS AND METHODS}

\section{Subjects}

The participants of the study were seven right-handed volunteers (four women, three men, mean age 29.1 years), who were psychostimulant-naïve. Subjects were medically fit and had no history of head injury and no current or past Axis I DSM-IV disorder, including substance abuse/ dependence on the basis of the Structured Clinical Interview for DSM-IV, clinician version (First et al, 1996). They had all been free of psychotropic medication for 1 year prior to the scan. Subjects were asked whether they had ever taken any psychotropic medication (ie medication to alleviate depression, anxiety, or other psychological disturbances). This was the case only for one subject who had taken psychotropic medication several years previous to the experiment. All subjects gave written informed consent for the study, which was approved by the local ethics committee.

\section{Experimental Design}

The study was a single-blind within-subjects design. Subjects received intravenous saline for a random period of $10-15 \mathrm{~min}$, followed by i.v. methamphetamine $(0.15 \mathrm{mg} /$ $\mathrm{kg}$ ) delivered over a $1 \mathrm{~min}$ period. The choice of this specific dose was based on pilot data obtained from drug-naïve volunteers where it was established as the smallest i.v. dose that reliably separated the effects of methamphetamine from placebo on subjective ratings. In addition, it is in line with previous studies (eg Kleinschmidt et al, 1999 who used a fixed dose of $15 \mathrm{mg}$ i.v. methamphetamine). The infusion was administered via an infusion pump that was located in a separate room. This enabled us to switch from saline to the active drug without the subjects being aware of this. They had been told that there was a $50 \%$ probability that they would receive the active drug. After the methamphetamine infusion, saline was restarted for a further $25-30 \mathrm{~min}$.

Functional images were obtained throughout the whole experiment, which lasted $41 \mathrm{~min}$ in total. Subjects rated themselves at 1-min intervals on a right-handed button press for the subjective experience of 'mind racing'. Ratings ranged from 0 (no mind racing) to 4 (high). Increases in this rating have been shown to correlate with the overall psychostimulant effect of methamphetamine and to be attenuated by dopamine depletion (McTavish et al, 1999; see also (Discussion'). 'Mind racing' was introduced and fully explained to each subject as follows: 'Mind racing is a condition you would not normally experience in day to day life. It is a state where your thoughts run far too quickly as if they were racing. One thought is replaced by another very quickly and it is difficult to hold on to your thoughts. You might have experienced a similar condition when you were very anxious or excited.' Following the scanning session, each participant was asked to describe their subjective experience. All subjects reported that they were well able to identify the subjective experience of mind racing as it occurred.

An arbitrary visual stimulus, the letter ' $\mathrm{L}$ ', was displayed on the screen during the experiment (including both the amphetamine and saline periods). Once a minute, the ratings were cued by another arbitrary visual stimulus, the letter ' $V$ ', which was displayed on a screen for $0.5 \mathrm{~s}$. This stimulus then changed back to ' $L$ ', which was shown for the remaining time.

\section{Image Acquisition}

Functional magnetic resonance (fMRI) images were acquired using a 3 Tesla Varian Inova MR imaging system. Each volume comprised $40 \mathrm{~T} 2{ }^{*}$-weighted contiguous axial slices (slice thickness $=6 \mathrm{~mm}$, slice gap $=0 \mathrm{~mm}$ ), acquired transversally using a single shot echo planar (EPI) pulse sequence $(\mathrm{TR}=5 \mathrm{~s})$. The in-plane field of view was $26.5 \times 26.5 \mathrm{~cm}^{2}$. The matrix dimensions were $64 \times 64$ with a voxel resolution of $4 \times 4 \mathrm{~mm}^{2}$ in-plane $\left(\right.$ a $8 \times 8 \mathrm{~mm}^{2}$ Gaussian filter was used for spatial smoothing). A reference whole-brain EPI scan was acquired for each subject. We used a set of optimizing techniques to select the imaging parameters in order to minimize susceptibility and distortion artifacts in the orbitofrontal cortex as described in detail by Wilson et al (2000). The relevant factors include minimizing voxel size in the plane of the imaging, as high a gradient switching frequency as possible $(960 \mathrm{~Hz})$, a short echo time of $25 \mathrm{~ms}$, and local shimming for the inferior frontal area.

\section{Data Analysis}

Imaging data were analyzed using SPM99 (Wellcome Department of Imaging Neuroscience, Institute of Neurology, London). Preprocessing of the data used SPM99 realignment and reslicing with sinc interpolation. FLIRT (FMRIB Centre, Oxford) was used to carry out intermodal registration and spatial normalization to the Montreal Neurological Institute (MNI)-brain (Collins et al, 1994). MNI coordinates were transformed into the Talairach coordinate system (Talairach and Tournoux, 1988) for description of the results. Spatial smoothing was applied with an $8 \mathrm{~mm}$ full-width at half-maximum isotropic Gaussian kernel. The time series at each voxel were highpass (cutoff period 650s) and low-pass filtered (by convolving the time series with a hemodynamic response kernel; Friston et al, 1994).

A general linear model was then applied to the time course of activation of each voxel. In order to investigate the brain activations related to amphetamine, three different models were constructed for each individual data set. An on-off model was designed to test for the main effects of amphetamine on the BOLD response. Based on the inspections of the changes in subjective ratings (see Figure 1), the 'on' period was modeled as an epoch having its onset $5 \mathrm{~min}$ after the start of the amphetamine injection and lasting for $10 \mathrm{~min}$. The 'off' epoch was the period during the initial saline infusion.

For the correlation analysis, the rating onsets were modeled as single impulse response functions (ie an event-related design) and then convolved with the canonical 
Mind-racing ratings $(0-4)$

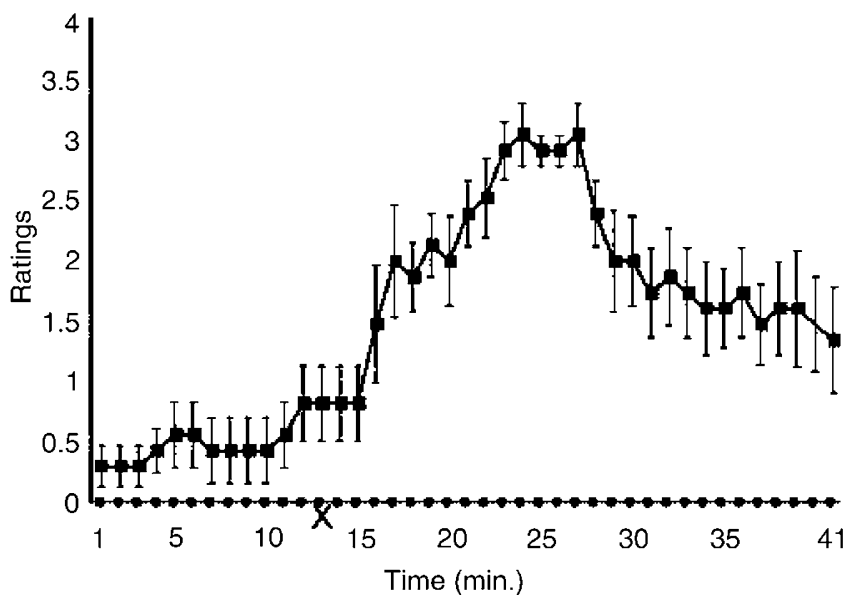

Figure I Averaged (mean \pm SEM) behavioral ('mind racing') ratings given by subjects across the experiment. The ' $X$ ' in the time axis shows the average time of the amphetamine infusion.

hemodynamic response function (HRF, Friston et al, 1994). The derivative of the HRF was included in the basis functions set. For each individual data set, the time series were linearly modulated by the behavioral ratings given by the subjects ('parametric modulation').

Linear contrasts of parameter estimates were then defined to test the specific effects of each condition within each individual data set. Voxel values for each contrast resulted in a statistical parametric map of the corresponding $t$ statistic, which was then transformed into the unit normal distribution (SPM Z). The statistical parametric maps from each individual data set were then entered into second-level, random effects analyses accounting for both scan-to-scan and subject-to-subject variability. The group effects of interest were assessed through applying one-sample $t$-tests to the first-level Z-maps.

Further, we were interested in studying the effects of methamphetamine in reward-related areas when no motorrelated responses were being given, to exclude the possibility that the activations observed resulted from an enhancement of central correlates of motor and related activity involved in making the ratings, including the button press, by amphetamine. The nonmotor related events of the task were (linearly) modeled in each individual data set by selecting a box-car basis function with onsets defined with a delay of $30 \mathrm{~s}$ with respect to the button press event (the ratings were given at every minute) and with a duration of $30 \mathrm{~s}$. The cutoff period for the high-pass temporal filter was set to $1200 \mathrm{~s}$, and no low-pass temporal filtering or global scaling was used. The value for the high-pass temporal filtering was designed in order to allow long-lasting, staterelated, effects of the drug to be revealed in the statistical analysis. This value also ensured that only within-subjects changes were modeled by the SPM analysis. A linear contrast was defined to test the comparison amphetamine epoch $v s$ saline epoch in the group analysis. These two time periods were defined using the timing frames described above for the 'on' and 'off periods. As for the other two models, the statistical parametric maps from each individual data set were entered into a second-level, random effects analysis and the group effects of interest were assessed through applying one-sample $t$-tests to the firstlevel Z-maps.

The present study reports $p$-values based on a group analysis for a priori regions of interest given the strong hypotheses generated by previous independent studies (Breiter and Rosen, 1999; Vollenweider et al, 1998; Goldstein and Volkow, 2002; Martinez et al, 2003; Volkow et al, $2001,2002)$. The $p$-values were corrected for the number of comparisons made within each region using the small volume correction (SVC) procedure (Worsley et al, 1996) consisting of defining a $10 \mathrm{~mm}$ sphere centered at the peak voxel of activation clusters. A priori regions of interest included the medial orbitofrontal cortex (Goldstein and Volkow, 2002; Volkow et al, 2001), the anterior cingulate cortex (Vollenweider et al, 1998; Volkow et al, 2002), and the (ventral) striatum (Breiter and Rosen, 1999; Martinez et al, 2003). Finally, checks were performed using the estimated motion as a covariate of no interest to rule out the possibility of the observed results being due to motionrelated artifacts.

\section{RESULTS}

\section{Behavioral Ratings}

All subjects tolerated the drug challenge without difficulty. The time course of subjective ratings, averaged across subjects (mean \pm SEM), is shown in Figure 1. The average onset of the amphetamine infusion (across subjects) is shown by the ' $\mathrm{X}$ ' symbol. All subjects experienced an increase in 'mind racing' within 2-3 min after the infusion, peaking at $10-15 \mathrm{~min}$ postinfusion. By the end of the experiment ratings were closer to baseline. Subjective reports taken from participants immediately after the experiment indicated that they experienced the subjective drug effects as positive and rewarding.

\section{Functional MRI Results}

Results for all activations are shown in Table 1.

On-off model. The comparison (Amphetamine-Saline) (see 'Materials and methods') produced significant activations in the medial part of the orbitofrontal cortex (Talairach coordinates [16 $38-8], Z=3.26, p<0.01$ SVC) and in the rostral part of the anterior cingulate cortex ([ $\left[\begin{array}{lll}-2 & 24 & -5\end{array}\right]$, $Z=3.19, p<0.01 \mathrm{SVC}$ ) where it borders ventrally the medial part of the orbitofrontal cortex. Less robust but significant activations were found in the right caudate nucleus where it borders the right putamen ([ $\left[\begin{array}{lll}16 & 18 & -9\end{array}\right], Z=2.79, p<0.05$ SVC). A further cluster of activation was observed in the right caudate nucleus ([ $\left[\begin{array}{lll}13 & 19 & 0\end{array}\right], Z=2.39, \quad p<0.005$ uncorrected). A cluster of activation was also found in the left motor cortex ([ $\left.\begin{array}{lll}-4 & -12 & 68\end{array}\right], \quad Z=2.89, \quad p<0.005$ uncorrected). The results are illustrated in Figure 2.

Correlation analysis. Correlation of the BOLD fMRI signal with individual mind-racing ratings (see 'Materials and methods') was significant in the caudate nucleus ([ $\left.\begin{array}{lll}5 & 15 & 1\end{array}\right]$, 
Table I Foci of Activation Clusters within Regions of Interest

Talairach coordinates of maximum activity

\begin{tabular}{|c|c|c|c|c|c|c|}
\hline \multirow[b]{2}{*}{ Brain region } & \multirow[b]{2}{*}{$x$} & & \multirow[b]{2}{*}{ Cluster size (voxels) } & \multirow[b]{2}{*}{ Z Score } & \multirow[b]{2}{*}{ p (SVC) } \\
\hline & & $y$ & $z$ & & & \\
\hline \multicolumn{7}{|l|}{ Amphetamine-Saline } \\
\hline Medial orbitofrontal cortex & 16 & 38 & -8 & 74 & 3.26 & 0.01 \\
\hline Rostral anterior cingulate & -2 & 24 & -5 & 44 & 3.19 & 0.01 \\
\hline Caudate/putamen & 16 & 18 & -9 & 16 & 2.79 & 0.05 \\
\hline Caudate nucleus & 13 & 19 & 0 & 8 & 2.39 & $0.005^{\mathrm{a}}$ \\
\hline \multicolumn{7}{|l|}{ Correlation analysis } \\
\hline Caudate nucleus & 5 & 15 & I & 103 & 3.38 & 0.005 \\
\hline Lateral orbitofrontal cortex & 39 & 44 & -8 & 10 & 2.81 & $0.01^{\mathrm{a}}$ \\
\hline Rostral anterior cingulate extending to medial OFC & 0 & 32 & -1 & 56 & 3.06 & 0.005 \\
\hline
\end{tabular}

ancorrected.

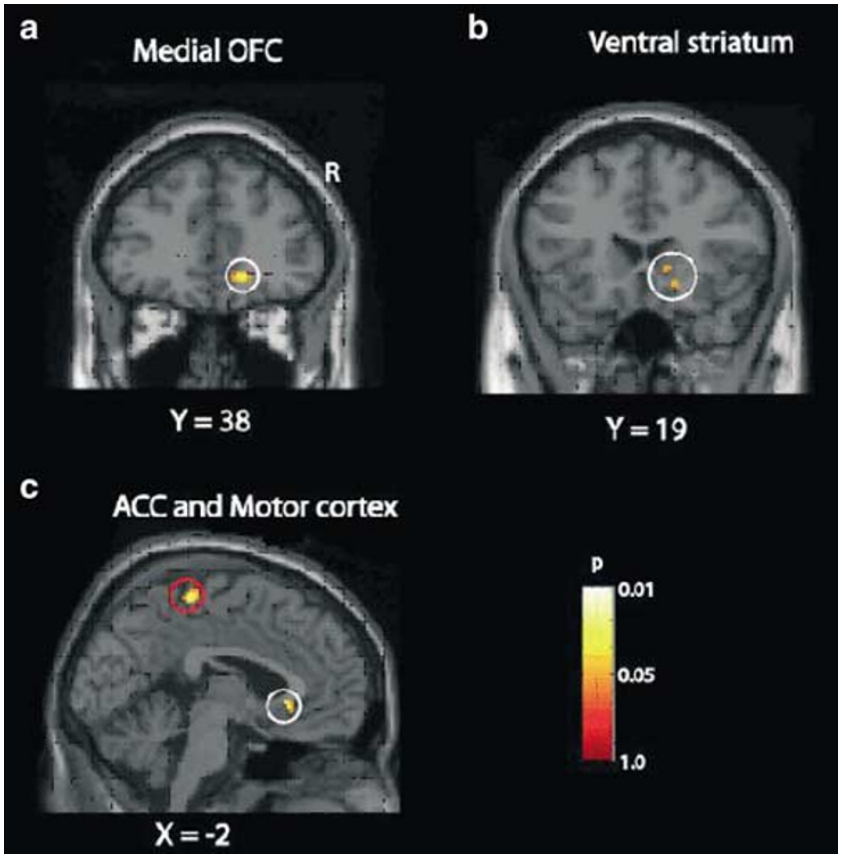

Figure 2 Results of the comparison Amphetamine-Saline. Activations (shown within the white circles) were found in (a) the medial orbitofrontal cortex, (b) ventral striatum, and (c) anterior cingulate cortex, and motor cortex (red circle). The $p$-values shown are with the small volume correction procedure (SVC, see 'Materials and methods').

$Z=3.38, p<0.005$ SVC) and in the rostral part of the anterior cingulate cortex $\left(\left[\begin{array}{lll}0 & 32 & -1\end{array}\right], Z=3.06\right.$, $p<0.005$ SVC). These activations are depicted in Figure 3 . At lower thresholds a significant correlation was found in the orbitofrontal cortex ([39 $44-8], Z=2.81, p<0.01$ uncorrected), and the rostral anterior cingulate activation just described extended into the medial orbitofrontal cortex.

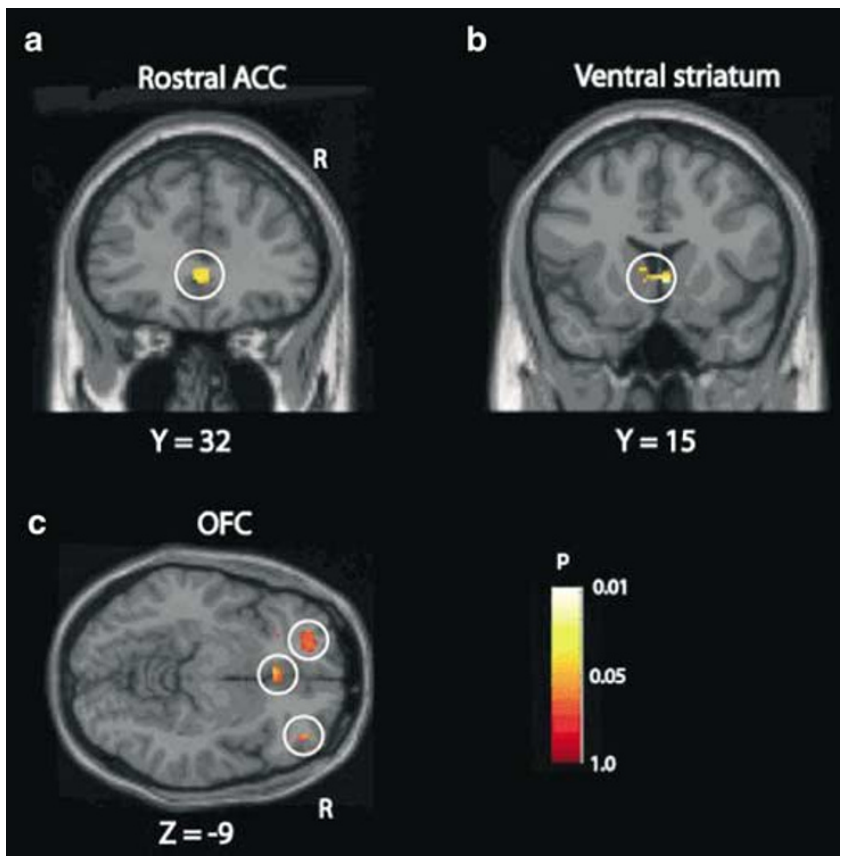

Figure 3 Results of the SPM analysis correlating changes in BOLD signal with subjective ratings of 'mind-racing' produced by methamphetamine. Correlated activations (shown within the white circles) were found in (a) the medial prefrontal cortex (rostral anterior cingulate cortex), (b) ventral striatum, and (c) orbitofrontal cortex. The p-values shown are with the small volume correction procedure (SVC, see 'Materials and methods').

Activations independent of motor response. Activations produced by amphetamine (as compared to saline), which were independent of the motor responses produced during the rating period (see 'Materials and Methods'), were found

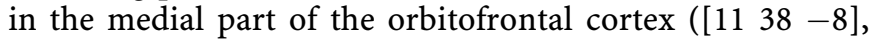
$Z=3.66, p<0.04$. SVC). This cluster of activation overlaps with the medial orbitofrontal cluster found to be activated in the comparison (Amphetamine-Saline) (see above). This 


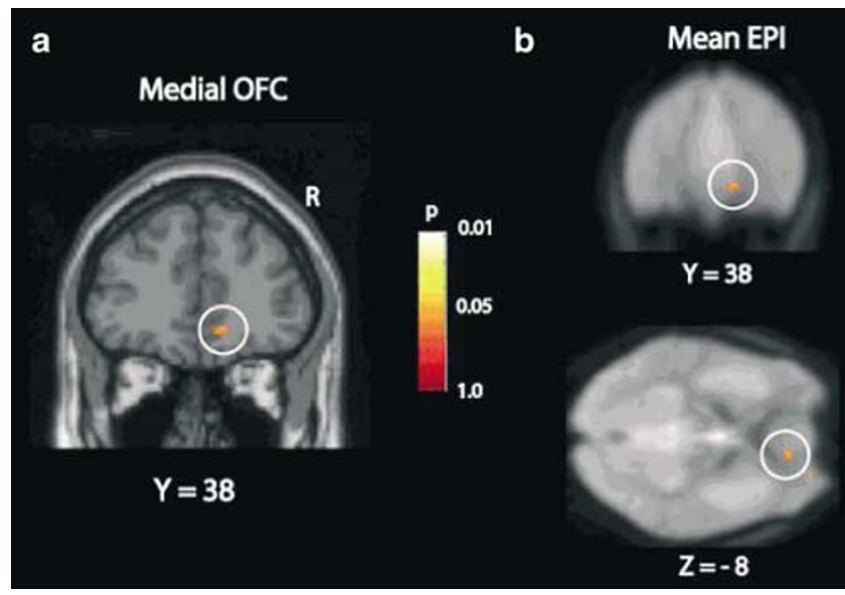

Figure 4 Activations in the medial orbitofrontal cortex (for the comparison (Amphetamine-Saline)) when no motor responses are being made. The most significant cluster of activated voxels for this comparison is shown. (a) The activation is shown superimposed on a high-resolution $\mathrm{TI}$ MRI coronal image. (b) The activation is shown superimposed on an EPI image obtained by averaging normalized mean EPI scans across all seven subjects. Coronal and axial images are shown. This illustrates good signal recovery in the orbitofrontal cortex region.

activation is shown in Figure 4, superimposed on a highresolution T1 MRI scan (and on the mean normalized EPI averaged across the seven subjects to show the good signal recovery in the orbital region).

Further analysis was carried out on this medial orbitofrontal cluster shown in Figure 4. The raw (time-series) signals from the voxels in this cluster were extracted and averaged across all the peristimulus time periods (ie the $1 \mathrm{~min}$ following a button press response) during the preinfusion (saline) and postinfusion (amphetamine) parts of the experiment. The average signals are shown in Figure 5(a). This graph demonstrates that this region of the orbitofrontal cortex was consistently activated by methamphetamine administration, whereas saline administration did not shift the signal from baseline. This increase was not dependent on motor responses: In Figure 5(b) we show the signal extracted from a motor cortex cluster (continuous with the cluster shown in Figure 2(c)), showing that in this case the activation is locked to the motor response that provided the ratings.

\section{DISCUSSION}

This study used fMRI to assess the responses in the human brain to intravenous administration of methamphetamine in amphetamine-naïve, healthy subjects. First, we showed that intravenous methamphetamine administration, when compared to saline administration, produces activity in reward- and affect-related areas of the human brain including the medial orbitofrontal cortex, the rostral anterior cingulate cortex and the (ventral) striatum. Second, activity in these areas was shown to correlate with the subjective ratings reflecting the effects of methamphetamine administration. Third, we have specifically shown that the orbitofrontal cortex is robustly activated by methamphetamine, in that increases in activation were observed across
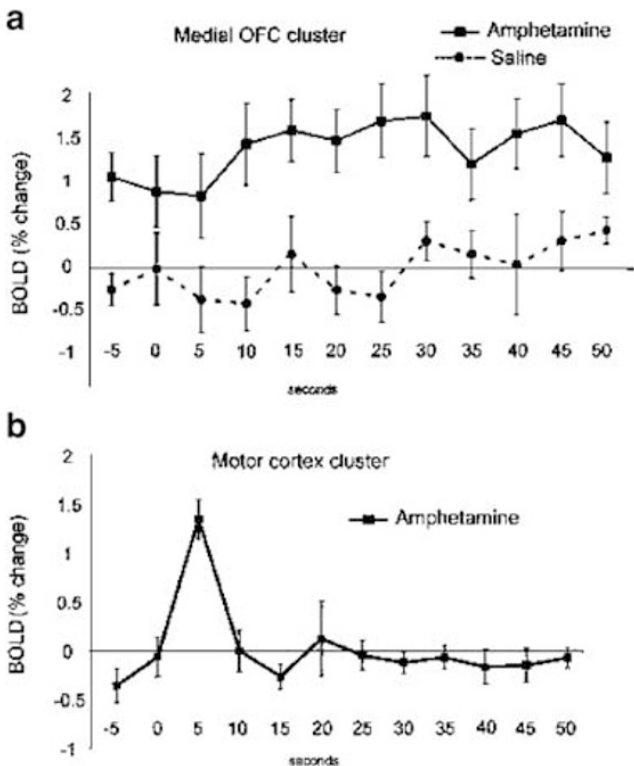

Figure 5 (a) $\mathrm{fMRl}$ time course of activations extracted from the orbitofrontal cortex cluster shown in Figure 4. The graph shows robust responses to amphetamine in this area in a way that is independent of the time of the button press made to give the rating at time 0 in the graph. No significant response in this region was produced during saline administration (prior to the amphetamine infusion). (b) Time course of activation extracted from a cluster in the motor cortex area illustrated in Figure 2(c), red circle. In this case, a robust brain activation follows the button press event in contrast to the time course shown in (a). No significant difference was found between the amphetamine and saline periods in this region.

the whole period following drug administration, and that these responses were not dependent on motor-related activity. This last finding provides evidence that the orbitofrontal cortex activation produced by methamphetamine is related to the nonphasic, nonmovement-related effects produced by amphetamine, which include effects such as mind racing, reward, and related effects that may be involved in the development of compulsive drug-intake and addiction (Volkow and Fowler, 2000; Volkow et al, 2001; Goldstein and Volkow, 2002).

Inspection of the behavioral data time courses indicates that the subjective ratings of 'mind-racing' did reflect the effects of amphetamine administration; this is in line with previous findings (McTavish et al, 1999). The cingulate and ventral striatum activations that correlated with these subjective experiences (see Figure 3) are in agreement with the PET study by Vollenweider et al (1998), where it was shown that $\mathrm{d}$-amphetamine produced a mania-like syndrome concomitantly with a widespread increase in absolute cerebral metabolism, which was significant in the anterior cingulate cortex, caudate nucleus, putamen, and thalamus. Dopamine release in the ventral striatum is linked to the rewarding and locomotor-stimulant effects of abused drugs such as amphetamine in experimental animals (Parkinson et al, 1999). In particular, conditioned reinforcement tasks are potentiated by amphetamine administration (Cardinal et al, 2002), and this potentiation is known to depend on dopamine innervation of the nucleus accumbens (Taylor and Robbins, 1986). In humans, this region was shown through fMRI to be activated by cocaine adminis- 
tration in cocaine abusers (Breiter et al, 1997; Breiter and Rosen, 1999).

The results described based on the correlation analysis are due largely to an effect of amphetamine on brain processes that occurred at about the time the ratings were given and the button press response was being made. Whether these effects of amphetamine are related to the cognitive evaluation of the current state of mind racing, or to the movements being made to press the button, is difficult to establish. To study brain activations that are independent of motor activity, we therefore used a model in which the event onsets were delayed with respect to the ratings. This analysis revealed a significant cluster of activation in the orbitofrontal cortex (Figure 4), which was not dependent on motor responses (as shown by the time course analysis illustrated in Figure 5). Activation in this area was found to be robust across the period following amphetamine injection, and was only revealed by the particular design used here. The fact that motor-independent activations were not found in other regions (such as the ventral striatum) could be for a number of reasons. One is that they may have been present, but were just not statistically significant. Another is that amphetamine increases activity in some of these regions (e.g. the ventral striatum, as shown in Figure 2) only in relation to the processes involved in giving the ratings such as motor movements.

The finding of significant orbitofrontal cortex activations independent of motor activity in drug-naïve subjects is of significance in the light of the proposed role of the orbitofrontal cortex in drug addiction (Volkow et al, 2001). The role of the primate orbitofrontal cortex in reward processing has been consistently established by neuronal recording studies (Rolls et al, 1989; Rolls, 1999, 2000). Evidence that, in humans, the orbitofrontal cortex is involved in reward processing comes from studies making use of a variety of stimuli, such as odor (Anderson et al, 2003; Rolls et al, 2003b), olfactory stimuli in a sensoryspecific satiety paradigm (O’Doherty et al, 2001), face attractiveness (O'Doherty et al, 2003), sexually arousing stimuli (Redoute et al, 2000), pleasant touch (Rolls et al, 2003a), consonant music sequences (Blood et al, 1999), food in the mouth when hungry (Kringelbach et al, 2003) and water in the mouth when thirsty (de Araujo et al, 2003). Based on these comparisons, we suggest that the activation of the orbitofrontal cortex by amphetamine reflects activation of one of the areas in the brain where amphetamine acts to produce rewarding effects and the associated subjective states. This is in line with the evidence that macaques will learn to self-administer very small quantities of amphetamine to this region (Phillips and Rolls, 1981), and that this is a region where electrical stimulation in macaques is rewarding (Rolls, 2000). This is also in line with the fact that the human orbitofrontal cortex region is in an area in which there are high concentrations of $D_{1}$ receptors (Hurd et al, 2001), and abnormal metabolic activity and low availability of dopamine $D_{2}$ receptors have been found in this area in the brain of drug addicts (Volkow et al, 2001).

In line with the above, the effects of amphetamine administration on human affective processing have been consistently established. In particular, a recent fMRI study by Hariri et al (2002) has shown that dextroamphetamine potentiated the amygdala response during the perceptual processing of angry and fearful facial expressions. This result is particularly interesting given the strong connectivity of the amygdala with the orbitofrontal cortex in primates (Rolls, 2000; Cavada et al, 2000). Cognitive processing, which depends on the prefrontal circuitry, can also be modulated by amphetamine administration. Of special interest is the fact that working memory performance is enhanced by amphetamine administration in subjects with relatively low working-memory capacity at baseline, whereas the reverse was found in high-capacity subjects (Mattay et al, 2000; Mattay et al, 2003).

Some limitations of the current study are worth mentioning. First, we used 'mind racing' for self-rating rather than other subjective drug effects that emphasize the emotional experience, such as 'rush', 'buzz' or 'euphoria'. It could be argued, therefore, that because of the behavioral measure used no inferences about the experience of reward can be made. However, 'mind-racing' has previously been shown to correlate with the overall psychostimulant effect of the drug in drug-naïve subjects (McTavish et al, 1999; McTavish et al, 2001). Specifically, McTavish et al (2001) showed this measure to be highly correlated with the rewarding experience of 'buzz'. These authors administered methamphetamine intravenously in a tyrosine depletion study in which subjective measurements of 'mind-racing', 'buzz', 'feeling good', 'alert', 'hunger', and 'depression' were collected. Only the measurements of 'buzz' and 'mindracing' were significantly attenuated by this dopaminedepleting treatment, of which 'mind-racing' was most significant. This suggests that 'mind-racing' may be the subjective experience most related to dopamine neurotransmission.

Furthermore, the attenuation of cognitive activationrelated increases in frontocortical regions under amphetamine (Gouzoulis-Mayfrank et al, 1999) goes against the conclusion that the activations we found represent correlates of cognitive phenomena only.

Second, the effects of methamphetamine administration on brain activity reported in this study are unlikely to be explained by peripheral changes caused by the drug such as heart-rate or respiratory changes. Part of the evidence for this is that only well-defined regions in the brain were found to be significantly activated by the drug, or to correlate with the 'mind racing' ratings. In addition, hemodilution effects related to intravenous drug administration would not explain the sharp onset of the effect after the amphetamine infusion, given that the experimental protocol starts with the saline administration. Furthermore, Kahn et al (1989) in a study with normal volunteers suggested decreases rather than increases in cerebral blood flow (measured by ${ }^{133} \mathrm{Xe}$ inhalation) occur after i.v. amphetamine despite increases in autonomic and behavioral arousal.

Finally, as subjective ratings were indicated by button press, in some brain areas the activations described could reflect a modulation by methamphetamine of the neural processes associated with cued finger movement. Uftring et al (2001) showed a significant increase in the number of activated voxels in the primary sensorimotor cortex during a simple motor task. However, on the basis of our analysis of the time course of activations in the orbitofrontal cortex, 
we could establish that the neuronal activations found in this area were not dependent on motor activity.

In conclusion, our findings support proposals implicating an important role of the orbitofrontal cortex in reward, particularly with respect to its putative role in drug addiction. As our subjects had no previous exposure to psychostimulants, our results are uncomplicated by phenomena such as craving and cued recall which would be present in drug-dependent individuals.

\section{ACKNOWLEDGEMENTS}

This research was supported by the Medical Research Council, UK.

\section{REFERENCES}

Anderson AK, Christoff K, Stappen I, Panitz D, Ghahremani DG, Glover $G$ et al (2003). Dissociated neural representations of intensity and valence in human olfaction. Nat Neurosci 6: 196-202.

Blood AJ, Zatorre RJ, Bermudez P, Evans AC (1999). Emotional responses to pleasant and unpleasant music correlate with activity in paralimbic brain regions. Nat Neurosci 2: 382-387.

Breiter HC, Rosen BR (1999). Functional magnetic resonance imaging of brain reward circuitry in the human. Ann NY Acad Sci 877: 523-547.

Breiter HC, Weisskoff RM, Kennedy DN, Makris N, Berke JD, Goodman JM et al (1997). Acute effects of cocaine on human brain activity and emotion. Neuron 19: 591-611.

Cardinal R, Parkinson JA, Hall J, Everitt BJ (2002). Emotion and motivation: the role of the amygdala, ventral striatum, and prefrontal cortex. Neurosci Biobehav Rev 26: 321-352.

Cavada C, Company T, Tejedor J, Cruz-Rizzolo RJ, Reinoso-Suarez $\mathrm{F}$ (2000). The anatomical connections of the macaque monkey orbitofrontal cortex. A review. Cereb Cortex 10: 220-242.

Collins DL, Neelin P, Peters TM, Evans AC (1994). Automatic 3D intersubject registration of MR volumetric data in standardized Talairach space. J Comput Assist Tomogr 18: 192-205.

de Araujo IE, Kringelbach ML, Rolls ET, McGlone F (2003). Human cortical responses to water in the mouth, and the effects of thirst. J Neurophysiol 90: 1865-1876.

First MB, Spitzer RL, Gibbon M, Williams JBW (1996). Structured Clinical Interview for DSM-IV Axis I Disorders, Clinician Version $(S C I D-C V)$. American Psychiatric Press: Washington, DC.

Friston K, Jezzard P, Turner R (1994). Analysis of functional MRI time-series. Hum Brain Mapp 1: 153-171.

Goldstein RZ, Volkow ND (2002). Drug addiction and its underlying neurobiological basis: neuroimaging evidence for the involvement of the frontal cortex. Am J Psychiatry 159: $1642-1652$.

Gouzoulis-Mayfrank E, Schreckenberger M, Sabri O, Arning D, Thelen B, Spitzer M et al (1999). Neurometabolic effects of psilocybin, 3,4-methylenedioxyethylamphetamine (MDE) and d-methamphetamine in healthy volunteers. A double-blind, placebo-controlled PET study with [18F]FDG. Neuropsychopharmacology 20: 565-581.

Hariri AR, Mattay VS, Tessitore A, Fera F, Smith WG, Weinberger DR (2002). Dextroamphetamine modulates the response of the human amygdala. Neuropsychopharmacology 27: 1036-1040.

Hurd YL, Suzuki M, Sedvall GC (2001). D1 and D2 dopamine receptor mRNA expression in whole hemisphere sections of the human brain. J Chem Neuroanat 22: 127-137.

Kahn DA, Prohovnik I, Luca LR, Sackeim (1989). Dissociated effects of amphetamine on arousal and cortical blood flow in humans. Biol Psychiatry 25: 755-767.
Kleinschmidt A, Bruhn H, Kruger G, Merboldt KD, Stoppe G, Frahm J (1999). Effects of sedation, stimulation, and placebo on cerebral blood oxygenation: a magnetic resonance neuroimaging study of psychotropic drug action. NMR Biomed 12: 286-292.

Kringelbach M, O'Doherty J, Rolls ET, Andrews C (2003). Activation of human orbitofrontal cortex to a liquid food stimulus is correlated with its subjective pleasantness. Cereb Cortex 13: 1064-1071.

McTavish SFB, McPherson MH, Harmer CJ, Clark L, Sharp T, Goodwin GM et al (2001). Antidopaminergic effects of dietary tyrosine depletion in healthy subjects and patients with manic illness. Br J Psychiatry 179: 356-360.

McTavish SFB, McPherson MH, Sharp T, Cowen PF (1999). Attenuation of some subjective effects of amphetamine following tyrosine depletion. J Psychopharmacol 13: 144-147.

Martinez D, Slifstein M, Broft A, Mawlawi O, Hwang DR, Huang Y et al (2003). Imaging human mesolimbic dopamine transmission with positron emission tomography. Part II: amphetamineinduced dopamine release in the functional subdivisions of the striatum. I Cereb Blood Flow Metab 23: 285-300.

Mattay VS, Callicott JH, Bertolino A, Heaton I, Frank JA, Coppola $\mathrm{R}$ et al (2000). Effects of dextroamphetamine on cognitive performance and cortical activation. Neuroimage 12: 268-275.

Mattay VS, Goldberg TE, Fera F, Hariri AR, Tessitore A, Egan MF et al (2003). Catechol $O$-methyltransferase val158-met genotype and individual variation in the brain response to amphetamine. Proc Natl Acad Sci USA 100: 6186-6191.

Murray JB (1998). Psychophysiological aspects of amphetamine abuse. J Psychol 132: 227-237.

O'Doherty J, Kringelbach ML, Rolls ET, Hornak J, Andrews C (2001). Abstract reward and punishment representations in the human orbitofrontal cortex. Nat Neurosci 4: 95-102.

O’Doherty J, Winston J, Critchley H, Perrett D, Burt DM, Dolan RJ (2003). Beauty in a smile: the role of medial orbitofrontal cortex in facial attractiveness. Neuropsychologia 41: 147-155.

Parkinson JA, Olmstead MC, Burns LH, Robbins TW, Everitt BJ (1999). Dissociation in effects of lesions of the nucleus accumbens core and shell on appetitive pavlovian approach behavior and the potentiation of conditioned reinforcement and locomotor activity by D-amphetamine. J Neurosci 19: 2401-2411.

Phillips AGMF, Rolls ET (1981). Intra-cerebral self-administration of amphetamine by rhesus monkeys. Neurosci Lett 24: 81-86.

Redoute J, Stoleru S, Gregoire MC, Costes N, Cinotti L, Lavenne F et al (2000). Brain processing of visual sexual stimuli in human males. Hum Brain Mapp 11: 162-177.

Rolls ET (1999). The Brain and Emotion. Oxford University Press: Oxford.

Rolls ET (2000). The orbitofrontal cortex and reward. Cereb Cortex 10: $284-294$.

Rolls ET, Kringelbach ML, de Araujo IE. (2003b). Different representations of pleasant and unpleasant odours in the human brain. Eur J Neurosci 18: 695-703.

Rolls ET, O’Doherty J, Kringelbach ML, Francis S, Bowtell R, McGlone F (2003a). Representations of pleasant and painful touch in the human orbitofrontal and cingulate cortices. Cereb Cortex 13: 308-317.

Rolls ET, Sienkiewicz ZJ, Yaxley S. (1989). Hunger modulates the responses to gustatory stimuli of single neurons in the caudolateral orbitofrontal cortex of the macaque monkey. Eur J Neurosci 1: 53-60.

Talairach J, Tournoux P (1988). Co-Planar Stereotaxic Atlas of the Human Brain. Stuttgart: Thieme.

Taylor JR, Robbins TW (1986). 6-Hydroxydopamine lesions of the nucleus accumbens, but not of the caudate nucleus, attenuate enhanced responding with reward-related stimuli produced by intra-accumbens D-amphetamine. Psychopharmacology 90: 390-397. 
Uftring SJ, Wachtel SR, Chu D, McCandless D, Levin DN, de Wit H (2001). An fMRI study of the effect of amphetamine on brain activity. Neuropsychopharmacology 25: 925-935.

Volkow ND, Chang L, Wang GJ, Fowler JS, Ding YS, Sedler M et al (2001). Low level of brain dopamine D2 receptors in methamphetamine abusers: association with metabolism in the orbitofrontal cortex. Am J Psychiatry 158: 2015-2021.

Volkow ND, Fowler JS (2000). Addiction, a disease of compulsion and drive: involvement of the orbitofrontal cortex. Cereb Cortex 10: $318-325$

Volkow ND, Fowler JS, Wang GJ, Goldstein RZ (2002). Role of dopamine, the frontal cortex and memory circuits in drug addiction: insight from imaging studies. Neurobiol Learn Mem 78: $610-624$.
Vollenweider FX, Maguire RP, Leenders KL, Mathys K, Angst J (1998). Effects of high amphetamine dose on mood and cerebral glucose metabolism in normal volunteers using positron emission tomography (PET). Psychiatry Res 83: 149-162.

Wilson JL, Jenkinson, de Araujo I, Kringelbach M, Rolls E, Jezzard P (2000). Fast, fully automated global and local magnetic field optimisation for fMRI of the human brain. Neuroimage 17: 967.

Woolverton WL, Cervo L, Johanson CE (1984). Effects of repeated methamphetamine administration on methamphetamine selfadministration in rhesus monkeys. Pharmacol Biochem Behav 21: 737-741.

Worsley KJ, Marrett P, Neelin AC, Friston KJ, Evans AC (1996). A unified statistical approach for determining significant signals in images of cerebral activation. Hum Brain Mapp 4: 58-73. 